\section{Shape-shifting materials}

Extreme deformation of particle rafts

How can we bend matter to our will? Folding patterns, as employed in origami, imply
severely limited shape-shifting severely limited shape-shifting possibilities. These soft materials also need to have the right shape-morphing properties to be useful for their National University has bee building and modelling particle rafts - new soft composite interfaces with superior deformability, that hold some future of wearable electronics, smart architecture.

olding is a process whereby an When folding objects like paper, often deliberately introduce creases, so the object will hold its new shape. With the right arrangement of folds, created with a whole host of different shapes and structures. A good paper for origami is thin enough
to be folded easily but sturdy enough that it will hold its shape and structure without bowing or tearing during the process. As paper is easy to crease, it is straightforward to give the material a 'memory' of the shape. In the case of paper, this process is irreversible, whic desired application. Professo
Ho-Young Kim at Seoul exciting possibilities for the may be problematic when it comes to reusing paper for future creations.

Biology and polymers do not initially seem to have much in common with the properties of paper. However, many bilogical systems are examples of soft, properties of polymers depend very much on their chemical compositio treatment, and processing, but they

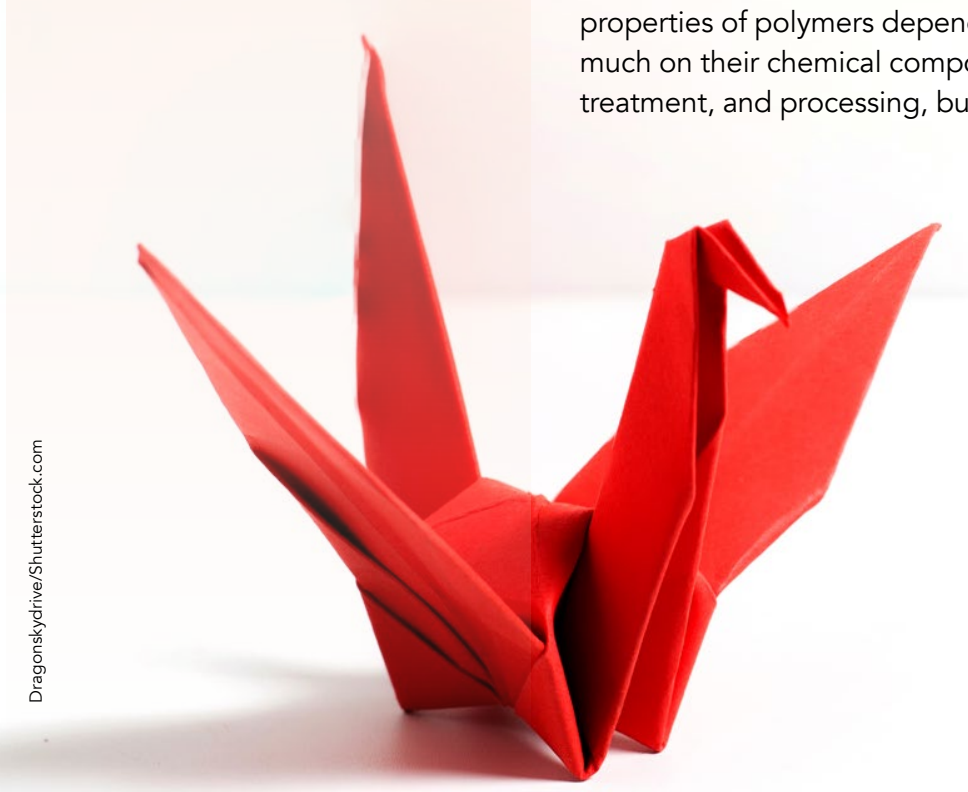

can also be designed to be highly elastic and flexible.

Creating materials that can be used as the starting point for shape morphing physical process of trying to fold paper First, there needs to be a way of manipulating and folding these soft systems at will. Often, the desired reshaping process is significantly more complex than simply folding the complex or substrate in half, so there needs to be a way to create the driving forces to form such shapes.

Nature already has many ways of achieving this level of shape morphing. One example is in plants, where parts of the plant structure can be arranged or swollen by transporting water to different areas. Some species, like mushrooms, have caps that start to droop in drier conditions as the water can deform the struct leaves, plants advantageous conditions, or, during the growth process, the growth can be driven asymmetrically to create the complex and often highly visually appealing construction of a flower peta.

Professor Ho-Young Kim at Seoul National University and his team have been puzzling over how to create artificia systems that can maintain some of the shape-morphing complexties found in natural species. As well as creating new materials that can be shaped at will into a variety of complex structures, Kim and his team have been working on new multiphysics models to explain the morphing process and the topography of the threewith their new soft ma they can create

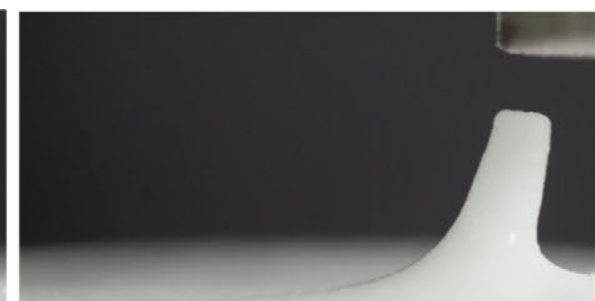

Electric-field-induced morphing of a particle raft.

\section{ELECTRIC FIELDS}

enge when designing a soft-matter system to undergo shape morphing is finding a way to trigger the shape-changing behaviour and control the geometry of the final state. Kim and strong electric fields.

Kim and his colleagues found that, on applying an electric field to a fluidfluid interface system, the
electric field acts electric field acts destabilise the fluid interfaces This can be a problem for some shape-

morphing materials as this mean for structures that generate high amounts of electric shear stresses near the interface, the overall stability of the structure is likely to be poor. If the interfacial deformation is extreme enough, this can lead to ejection, detachment, and jetting of the particles, which can completely

\section{DESIGN TRICKS}

To avoid any issues with electric shear stresses, Kim and the team designed their new material to include a raft of dry insulating particles at the interface and a balance mechanism arising from electric discharge. This means no extreme shear stresses build up that would result in the destruction of the that the particles remain stablures

Controllable, deformable soft-matter materials, like Professor Kim's, could potentially be used to create flexible and smart structures.

they have undergone the morph into their new shape.

Making Kim's new particle rafts meant designing a material that was highly elastic. This meant the shape-changing process could be reversible and controllable. Unlike with folding paper, creases, these flexible softmatter systr can be transformed between different That has meant designing amounts of asymmetric forces but that can keep a smooth and stable surface

The new materials designed by Kim and his team can be morphed into towerelectrode. The towers can also be translated by moving the electrode. While this material looks incredibly promising in terms of applications controllable soft matter, there are still many more questions to answer about some of the dynamic and non-linear behaviours of the materials more generally. The team believe the rafts are ideal model two-dimensional granular systems, and they are som of the first systems that can also be propelled horizontally wh

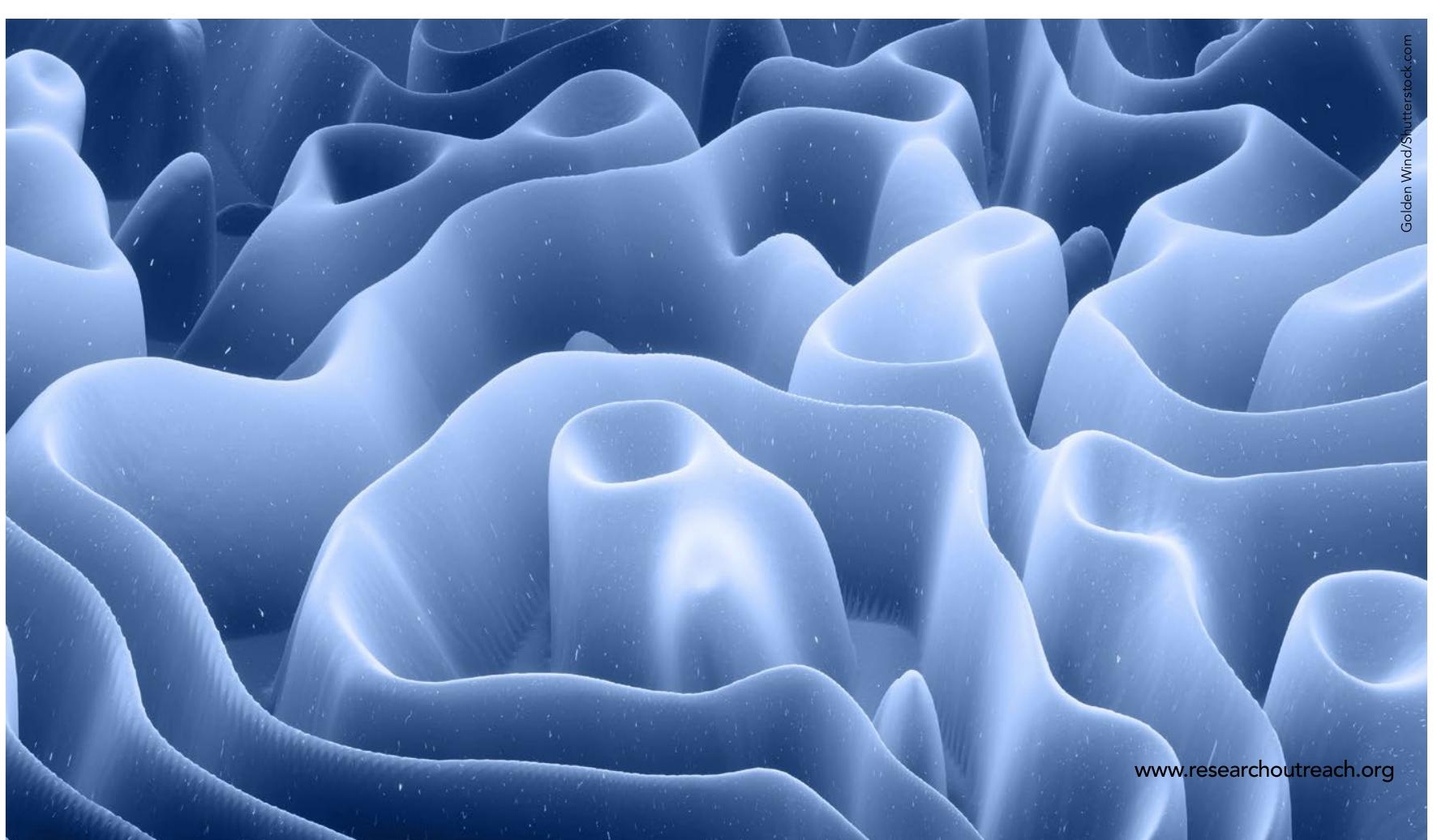




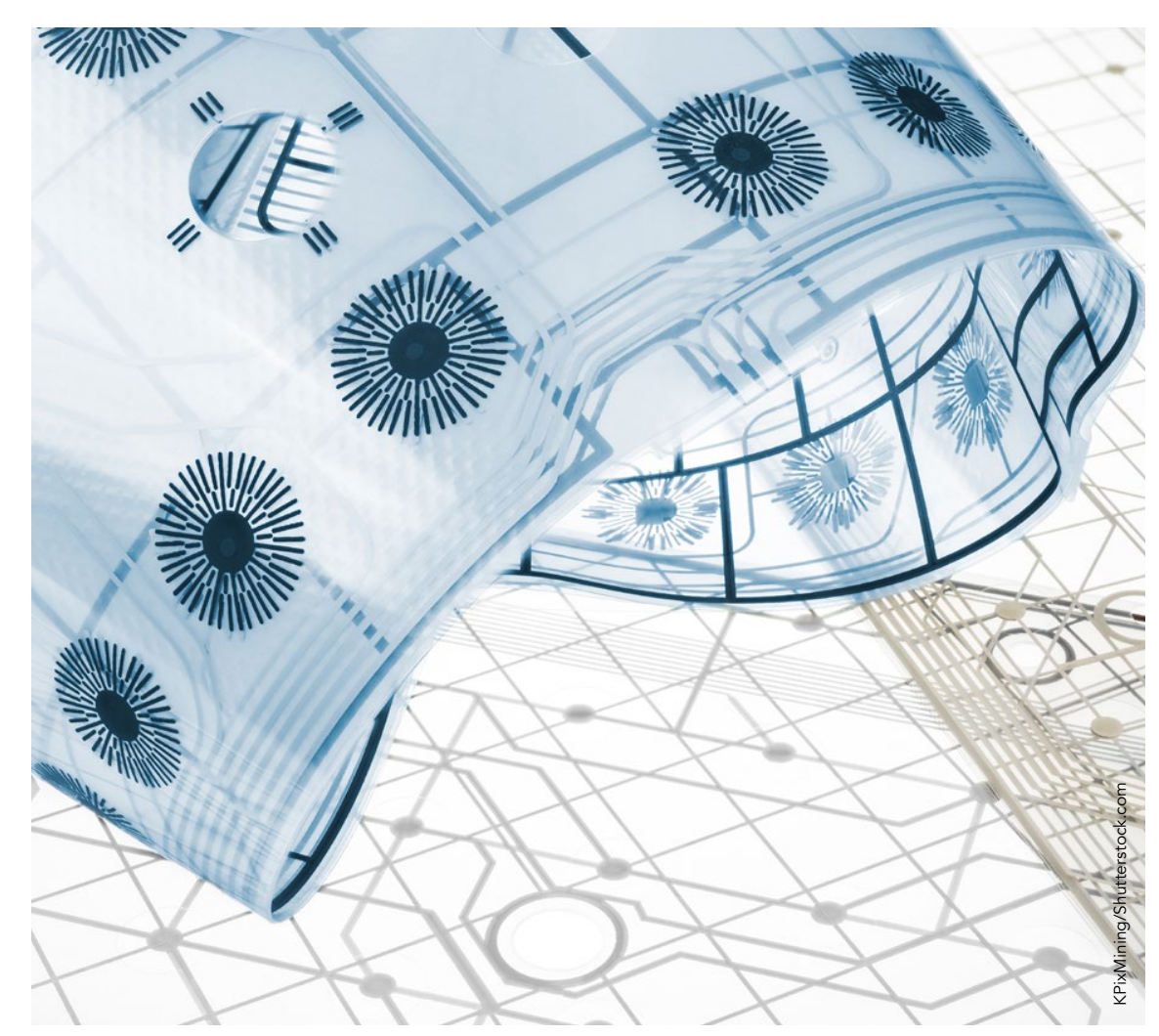

These materials enable the rese

It has been a huge challenge to find particle
systems systems This material looks incredibly promising in terms of applications that demand a controllable soft matter.

create flexible and smart structures. As the morphing process is reversible with is just a case of finding sources to act as electrodes to provide the necessary fields for the shape-changing to take place. One possibility for this could be human fingers or other polymer species to start to develop controllable human-machine interfaces. Otherwise, they could be used to create more advanced articulation in robots for more complex tasks than can be achieved with standard mechanical articulation.

Other possibilities exist in the world of biomedicine. Stretchable, elastic polymers could be used to create artificial, selfhealing skin. Another use of morphable particle systems could be in drug delivery the material sructure and can then be trection the of ensuring drugs are delivered ony t the required site, and a good way

assembled into illable softher regions
of the body. three-dimensional shapes and remain stable, let alone to find one that can also be propelled. Kim's work represents a huge step forward, not only in better understanding the complexities of these Controllable, deformable soft materials,
morphable soft-matter systems, but also like Kim's, could potentially be used to of minimising or unwanted uptake of drugs

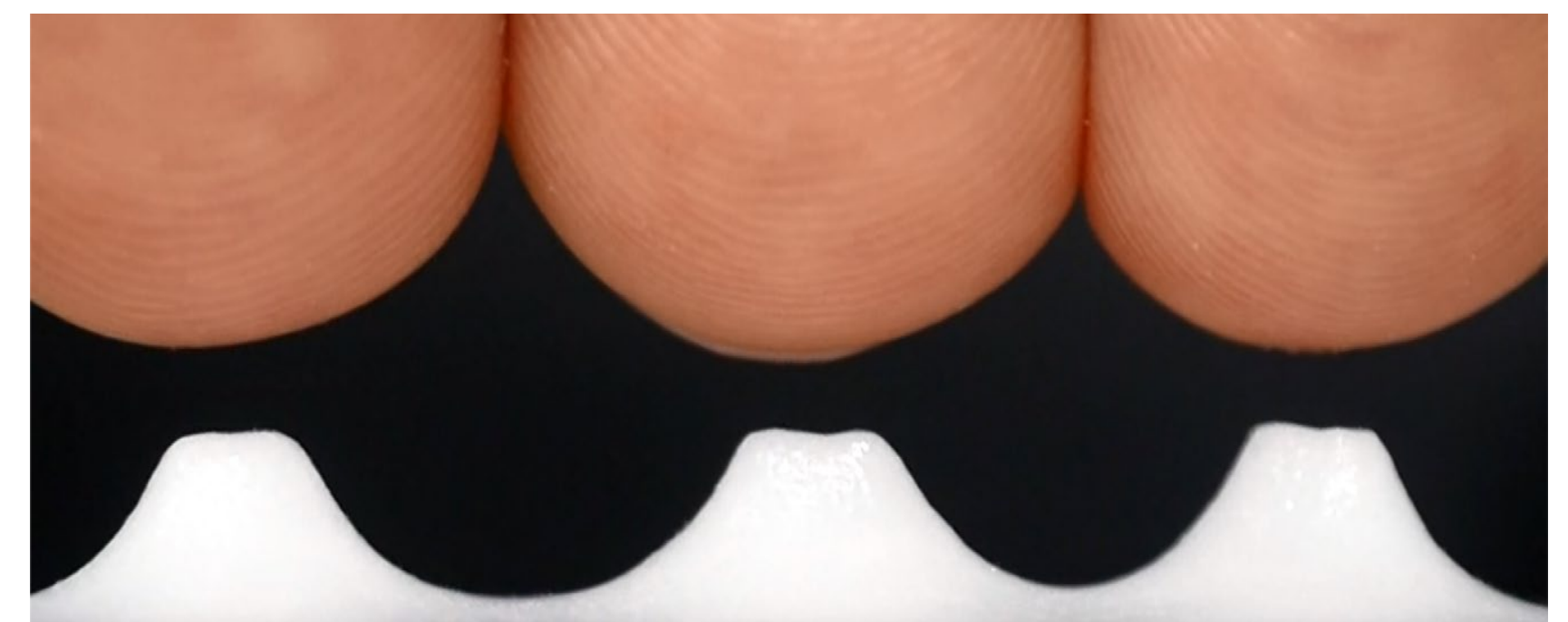

$5 \mathrm{~mm}$ applications.

Kim's material has a promising future and his multi-physics models ather researc step in helping other researchers design flexible, foldable systems for the soft matter APPLICATIONS OF SOFT MATERIALS like Kim's, could potentially be used to of the future. where the drug is encapsulated into

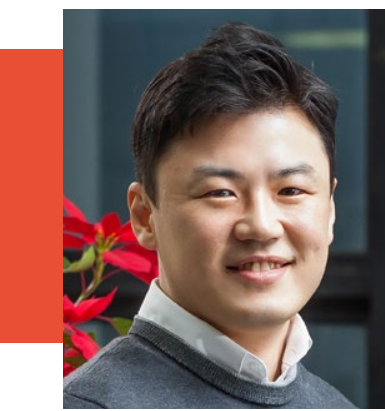

\section{Behind the Research}

\section{Professor Ho-Young Kim}

E: hyk@snu.ac.kr T: $+8228809286 \quad$ W: fluids.snu.ac.kr
Research Objectives

Professor Ho-Young Kim at Seoul National University has been building and modelling particle rafts.

\section{Detail}

\section{Address}

Department of Mechanical Engineering, Seoul National

University, Seoul 08826, Korea

Bio

Ho-Young Kim is a Professor in the Department of

Mechanical Engineering at Seoul National University. He

received his PhD in Mechanical Engineering at MIT and was

His current research interests are in soft matter dynamics,

microfluid mechanics, biomimetics and soft robotics.

Funding

earch Foundation of Korea

(grant no. 2018-052541)

Collaborators

- Mr Kyungmin Son

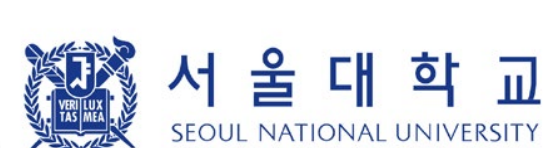

elected a Fellow of the American Physical Society in 2017.

\section{5}

\section{3

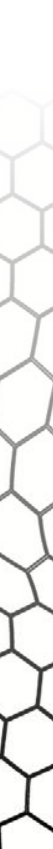

\section{References}

Son, K, Sun, J-Y, Kim, H-Y, (2021) Agile reversible shapemorhphing of particle rafts, Soft Matter, 17, 7554-64. do org/10.1039/d1sm00564b

Guzowski, J, Gim, B, (2019) Particle clusters at fluid-fluid interfaces: equilibrium profiles, structural mechanics and org/10.1039/c9sm00425

Siéfert, E, Reyssat, E, Bico, J, Roman, B, (2019) Bio-inspired pneumatic shape-morphing elastomers. Nature Materials, 18 , 24-29. doi.org/10.1038/s41563-018-0219-x

Collins, RT, Jones, JJ, Harris, MT, Basaran, OA, (2007) Electrohydrodynamic tip streaming and emission of charged drops from liquid cones. Nature Physics, 3, 149-154. do. org/10.1038/nphys807

Vella, D, Aussillous, P, Mahadevan, L, (2004) Elasticity of an interfacial particle raft. Europhysics Letters, 68, 212-218. doi. org/10.1209/epl/i2004-10202-x

\section{Personal Response}

What types of structure do you think it may be possible to create in the future?

II By sophisticated control of the external electric complicated three-dime to face, and to build tall and complex structures like a miniature Eiffel Tower. This will eventually lead to a threerevolutionise how we create and consume visual contents
in the coming era of the metaverse. stability agahist detachment Sotter, 15, 4921-38. doi dimensional physical display with high precision, which will

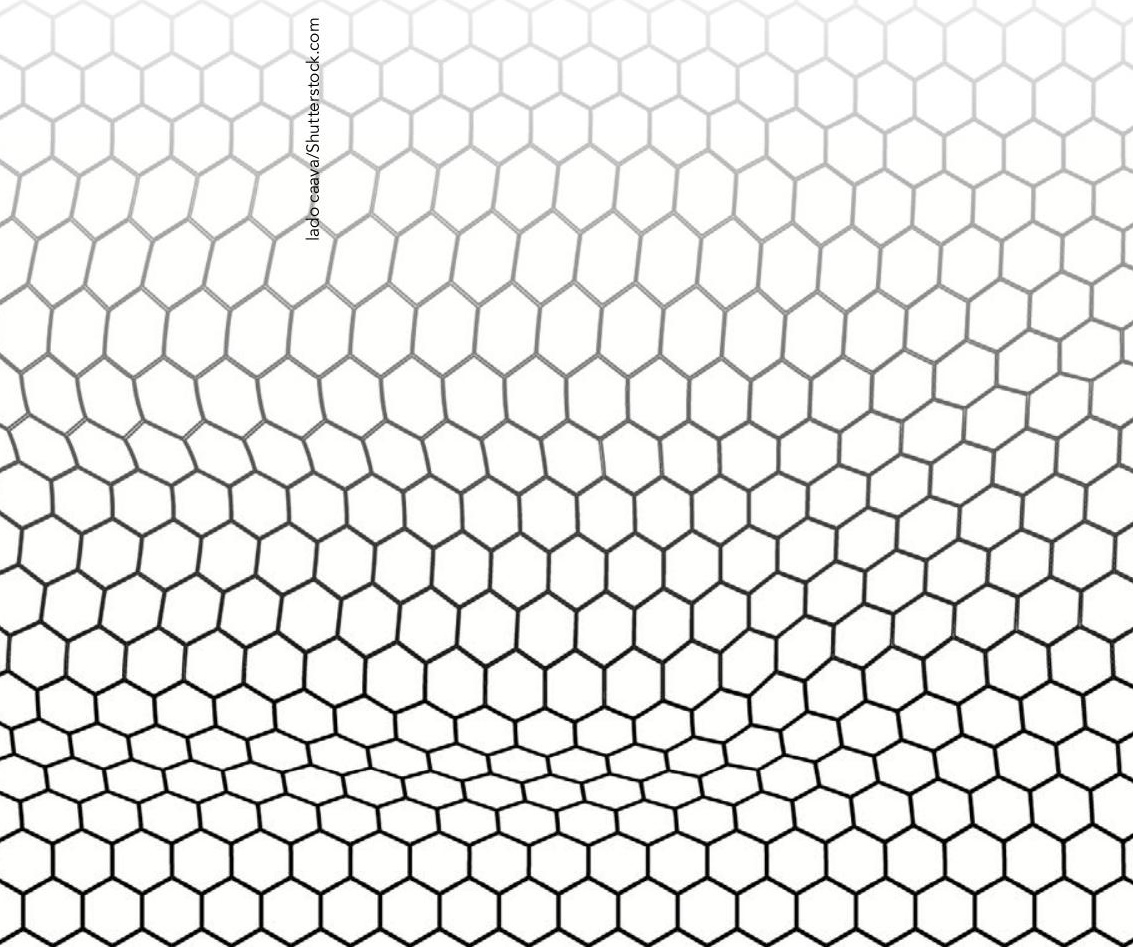

